\title{
Case Report \\ Sarcoidosis Incidentally Diagnosed: A Case Report
}

\author{
Besir Kesici, ${ }^{1}$ Ahmet Burak Toros, ${ }^{1}$ Levent Bayraktar, ${ }^{2}$ and Adem Dervisoglu ${ }^{3}$ \\ ${ }^{1}$ Department of Gastroenterohepatology, Medical Faculty Bahcesehir University, Istanbul, Turkey \\ ${ }^{2}$ Department of Radiology, Medical Park Fatih Hospital, Istanbul, Turkey \\ ${ }^{3}$ Department of General Surgery, Medical Faculty Bahcesehir University, Istanbul, Turkey
}

Correspondence should be addressed to Ahmet Burak Toros; aburaktoros@yahoo.com

Received 9 May 2014; Accepted 1 July 2014; Published 8 July 2014

Academic Editor: Coline Van Moorsel

Copyright (C) 2014 Besir Kesici et al. This is an open access article distributed under the Creative Commons Attribution License, which permits unrestricted use, distribution, and reproduction in any medium, provided the original work is properly cited.

\begin{abstract}
Sarcoidosis is a chronic, granulomatous condition with unknown cause. Because most of the patients are free of clinical symptoms, sarcoidosis should be considered in differential diagnosis if noncaseous granuloma is noted in biopsies, performed for other reasons. With no clinical symptoms, our patient was diagnosed with sarcoidosis upon identifying noncaseous granuloma in the lymph node biopsy material collected during the laparoscopic operation, performed for gallbladder polyp.
\end{abstract}

\section{Introduction}

Sarcoidosis can be seen in all races, at all ages, and in both sexes. Its prevalence varies by the organs involved, severity of the disease, and clinical course, as well as among populations and races $[1,2]$. The prevalence is higher in Afro-Americans and in the Scandinavian race. The incidence of sarcoidosis in Turkey is estimated to be $4 / 100.000$ [3] Etiological factors leading to sarcoidosis are not clear yet [1]. The typical histopathologic lesion of sarcoidosis is granuloma without caseous necrosis [2]. Almost half of the patients with sarcoidosis present with no symptoms. Diagnosis in some cases is made with the help of findings observed in chest $\mathrm{X}$-ray taken for other reasons. Since the lungs are the most frequently involved organ (90-95\%), patients usually present with pulmonary complaints (e.g., shortness of breath, dry cough) $[1,2,4]$. Some cases, on the other hand, might be seeking medical attention for constitutional symptoms or signs of extrapulmonary site involvement [1]. One-third of the cases may present with nonspecific complaints such as fatigue, weakness, easy tiredness, and weight loss. Nocturnal sweating may rarely be present. Fever may persist for weeks and be $40^{\circ} \mathrm{C}$ in rare cases [4]. Diagnosis is based on pathologic demonstration of granulomas without caseous necrosis in the presence of clinical and radiological findings consistent with sarcoidosis, where other causes which might be associated with a similar manifestation are ruled out $[1,4]$.

\section{Case Report}

The 20-year-old female patient presented with abdominal pain, which began 10 days ago, and underwent relevant examinations, among which abdominal USG demonstrated a polypoid lesion with a diameter of $5.7 \mathrm{~mm}$ in the gallbladder, and laparoscopic cholecystectomy was therefore performed. Plaques, white in color, on the liver were noted during the surgery, although biopsy material was not collected, due to the risk of bleeding. The patient was referred to our clinic when chronic cholecystitis and nonnecrotizing granulomatous lymphadenitis in one lymph node were identified in the sections. Patient's investigations yielded normal hepatic enzymes; HBsAg and AntiHCV were negative. PA chest X-ray demonstrated fullness at both hili. Thoracic CT was performed (Figures 2, 3, 4, and 5) and was reported as upper mediastinal-bilateral superior and inferior paratrachealaortopulmonary-paraesophageal-subcranial LAMs, the largest of which has a diameter of up to $13 \mathrm{~mm}$, are observed. Pleuroparenchymal sequelae densities are present in the apical lobe of bilateral lungs. Centrilobular nodules are observed at bilateral lungs. There are celiac-periportal LAMs in the section crossing the upper abdomen. The findings reported principally suggest sarcoidosis. Patient's biopsies were therefore reevaluated at the pathology clinic of the university hospital. Nonnecrotizing granulomatous lymphadenitis was identified at the lymph node. The findings suggested sarcoidosis. Increased levels of angiotensin-converting enzyme 
(ACE) were found (197,5 U/L; normal range: 8-52). Calcium level was normal.

\section{Discussion}

Sarcoidosis is a chronic, granulomatous condition with unknown cause [5]. Diagnosis is based on tissue biopsies, demonstrating granulomas without caseous necrosis in the presence of clinical and radiological findings, consistent with sarcoidosis, where other causes which might be associated with a similar manifestation are ruled out $[1,4]$. Presence of granulomas alone is not diagnostic. From the histological perspective, same lesions may also be observed in chronic berylliosis, tuberculosis, histoplasmosis, coccidioidomycosis, lymphoma, Hodgkin's disease, bronchogenic carcinoma, foreign body granuloma, schistosomiasis, syphilis, and leprosy [6].

Clinical signs depend on disease duration, organ involvement, extent of involvement, and the activity of the granulomatous event.

Lungs are the most frequently involved organ. Half of the patients are asymptomatic and are usually diagnosed when investigating for another cause. Bilateral hilar lymphadenopathy is characteristic and usually accompanied by paratracheal lymphadenopathy [7]. Our patient was diagnosed upon identifying noncaseous granuloma in the lymph node biopsy material, collected during the operation performed for gallbladder polyp.

Serum ACE is increased in 30 to $80 \%$ of patients with sarcoidosis [8]. This variation is considered to be associated with measurements performed at different timeframes and different radiological stages as well as with the presence of extrapulmonary lesions $[9,10]$. There are contradicting data on the disease and activity relationship. In our patient, the disease was clinically inactive despite the increased ACE level.

Although hepatic involvement is seen in $75 \%$ of the cases in sarcoidosis, clinical symptoms and impairment of the hepatic function are not common [11]. Plaques forming in the liver usually go unnoticed with imaging techniques. The nodules, which in rare cases reach to a diameter of $2 \mathrm{~cm}$, may be mistaken for metastasis [12]. Similarly, white plaques were noted on the liver surface during our patient's surgery (Figure 1). On the other hand, liver was in normal appearance with abdominal USG and CT.

Sarcoidosis has a variable clinical course and prognosis. One-third of the patients undergo spontaneous remission. In $10-30 \%$ of the patients, however, the disease takes a chronic course $[13,14]$. Corticosteroids remain as the main therapeutic choice [15]. Because our patient had no clinically relevant complaints, it was decided to follow up the patient without treatment (in accordance with a pulmonologist, who also performed on the patient a breath function test).

\section{Conflict of Interests}

The authors declare that there is no conflict of interests regarding the publication of this paper.

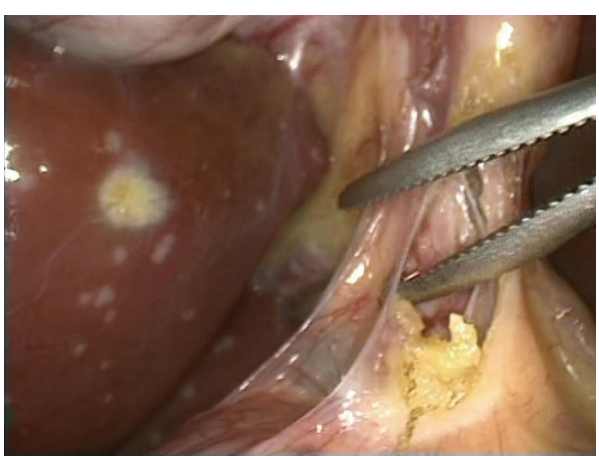

FIgURE 1: White plaques on the liver, noted during laparoscopy.

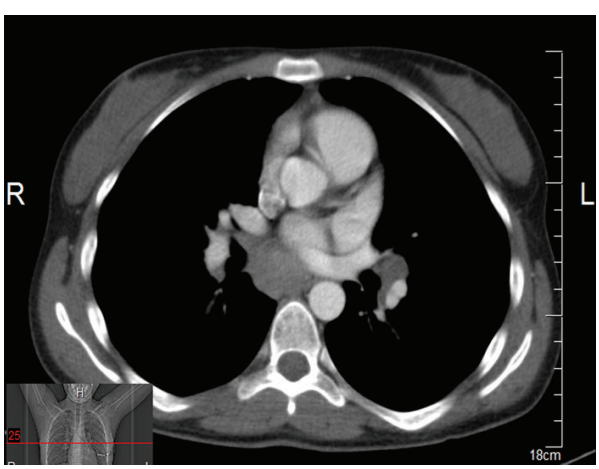

Figure 2: Thoracic CT views.

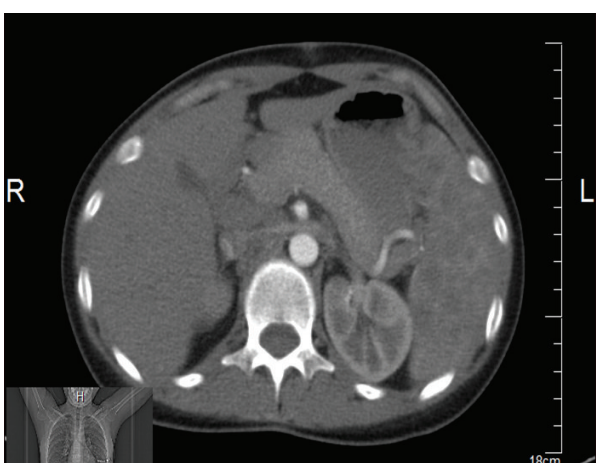

FIGURE 3: Thoracic CT views.

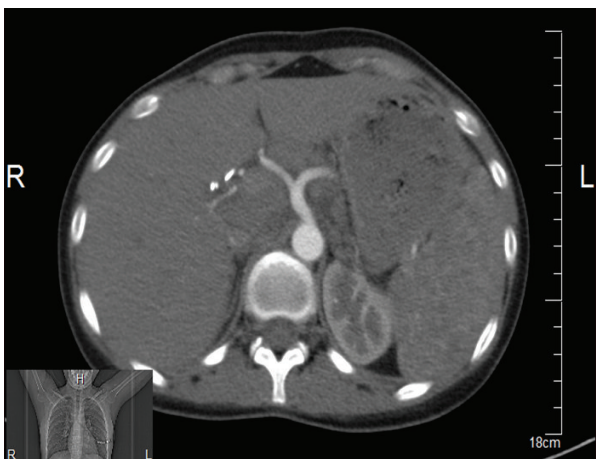

FIgURE 4: Thoracic CT views. 


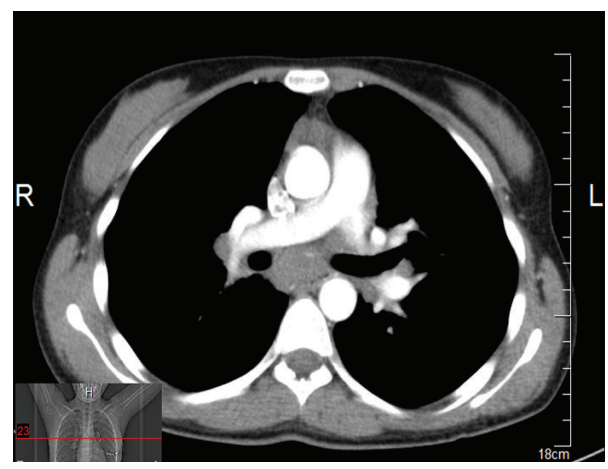

Figure 5: Thoracic CT views.
[11] T. B. Reynolds, J. L. Campra, and R. L. Peters, "Granulomatous liver disease," in Hepatology, D. Zakim and T. D. Boyet, Eds., vol. 2, Saunders, Philadelphia, Pa, USA, 3rd edition, 1996.

[12] J. Farman, G. Ramirez, J. Brunetti, J. Tuvia, C. Ng, and H. Rotterdam, "Abdominal manifestations of sarcoidosis CT appearances," Clinical Imaging, vol. 19, no. 1, pp. 30-33, 1995.

[13] A. Baran, F. Özşeker, D. Güneylioğlu et al., "Sarcoidosis: yedi yıllık deneyim," Toraks Dergisi, vol. 5, pp. 160-165, 2004.

[14] P. L. Joseph, A. K. Ella, and E. G. Stevan, "Pulmonary sarcoidosis," Clinics in Chest Medicine, vol. 18, no. 4, pp. 755-785, 1997.

[15] L. S. Newman, C. S. Rose, and L. A. Maier, "Sarcoidosis," The New England Journal of Medicine, vol. 336, no. 17, pp. 1224-1234, 1997.

\section{Acknowledgment}

This paper has been seen and approved by all authors; it is an original contribution not previously published; it is not under consideration for publication elsewhere. The authors received no financial support.

\section{References}

[1] "Statement on sarcoidosis. Joint Statement of the American Thoracic Society (ATS), the European Respiratory Society (ERS) and the World Association of Sarcoidosis and Other Granulomatous Disorders (WASOG) adopted by the ATS Board of Directors and by the ERS Executive Committee, February 1999," American Journal of Respiratory and Critical Care Medicine, vol. 160, no. 2, pp. 736-755, 1999.

[2] G. W. Hunninghake, U. Costabel, M. Ando et al., "ATS/ERS/WASOG statement on sarcoidosis," Sarcoidosis Vasculitis and Diffuse Lung Disease, vol. 16, no. 2, pp. 149-173, 1999.

[3] B. Musellim, O. O. Kumbasar, G. Ongen et al., "Epidemiological features of Turkish patients with sarcoidosis," Respiratory Medicine, vol. 103, no. 6, pp. 907-912, 2009.

[4] M. C. Lannuzzi, B. A. Rybicki, and A. S. Teirstein, "Sarcoidosis," The New England Journal of Medicine, vol. 357, pp. 2153-2165, 2007.

[5] M. Ng and J. K. Niparko, "Osseous sarcoidosis presenting as a destructive petrous apex lesion," American Journal of Otolaryngology, vol. 23, no. 4, pp. 241-245, 2002.

[6] D. N. Mitchell and J. G. Scadding, "Sarcoidosis," American Review of Respiratory Disease Journal, vol. 110, no. 6, pp. 774$802,1974$.

[7] K. Ellis and G. Renthal, "Pulmonary sarcoidosis. Roentgenographic observations on course of disease," American Journal of Roentgenology, vol. 88, pp. 1070-1083, 1962.

[8] L. T. Tanoue and J. A. Elias, "Systemic sarcoidosis," in Textbook of Pulmonary Disease, G. L. InBaum, J. D. Crapo, B. R. Celi, and J. B. Karlinsky, Eds., pp. 407-430, Lippincott-Raven, Philadelphia, Pa, USA, 6th edition, 1998.

[9] P. Sharma Om, “Sarcoidosis," Disease-a-Month, pp. 417-452, 1990.

[10] B. Podwysocki, S. Skowron-Szlósarczyk, J. Zwoliński, E. Szklarz, E. Jankowska, and M. Masiak, "The usefulness of serum angiotensin-converting enzyme test in the diagnosis of sarcoidosis.", Materia Medica Polona, vol. 23, no. 2, pp. 121-124, 1991. 


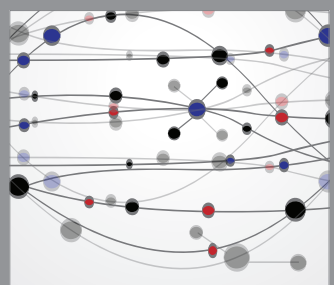

The Scientific World Journal
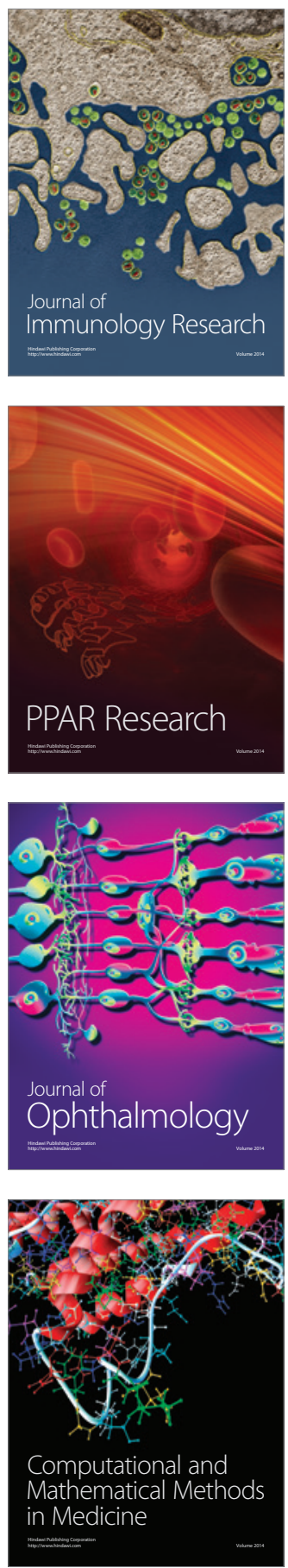

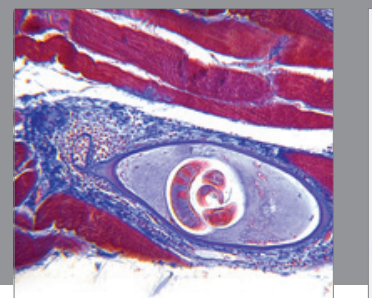

Gastroenterology

Research and Practice
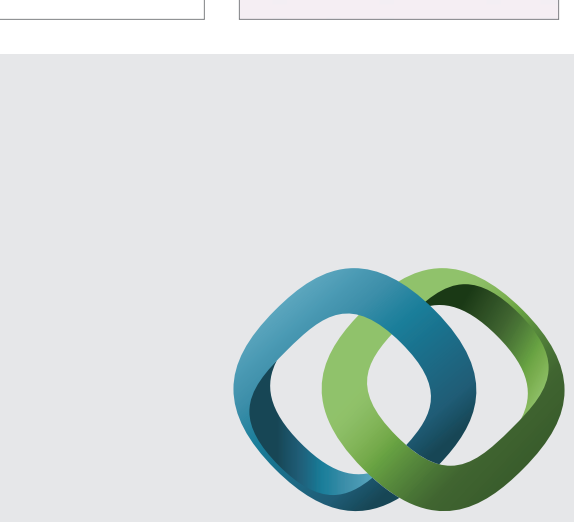

\section{Hindawi}

Submit your manuscripts at

http://www.hindawi.com
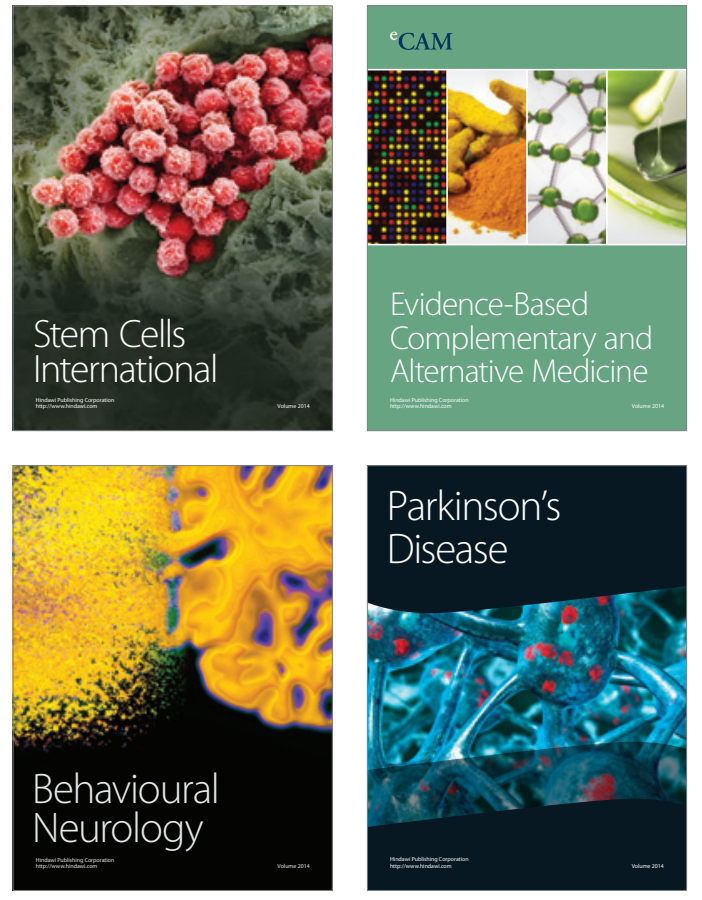
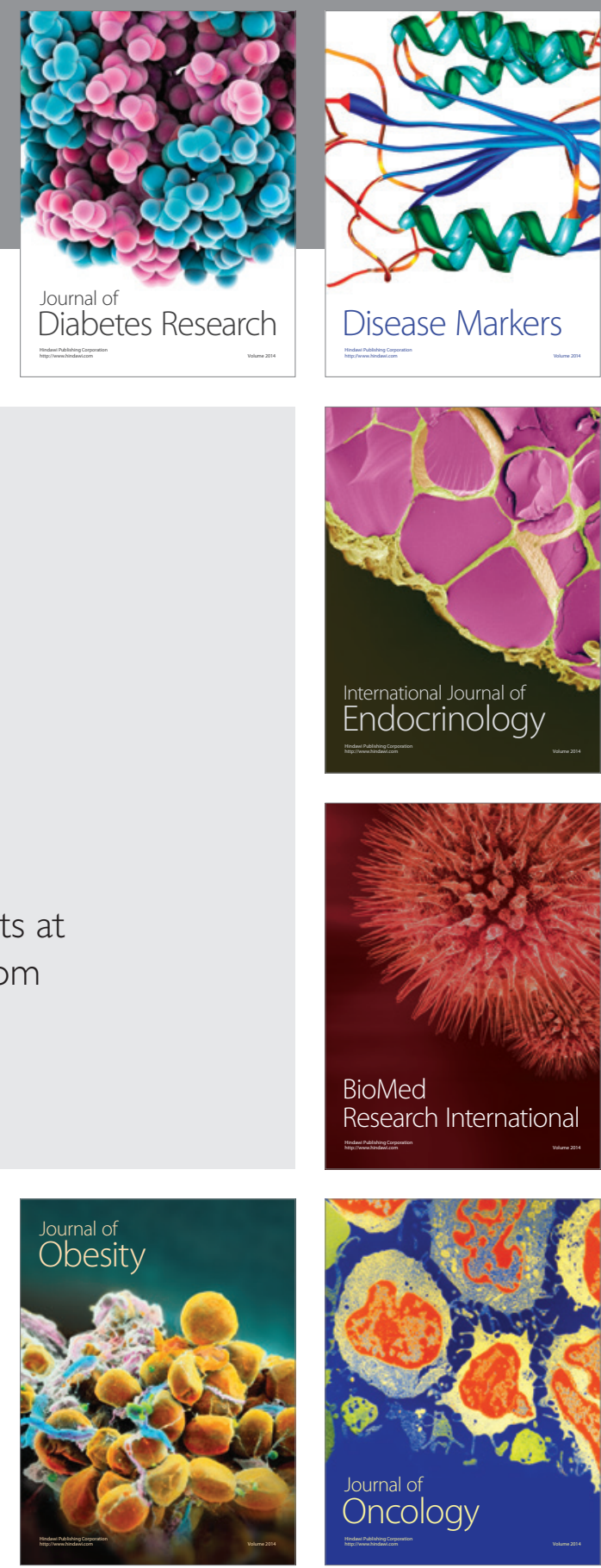

Disease Markers
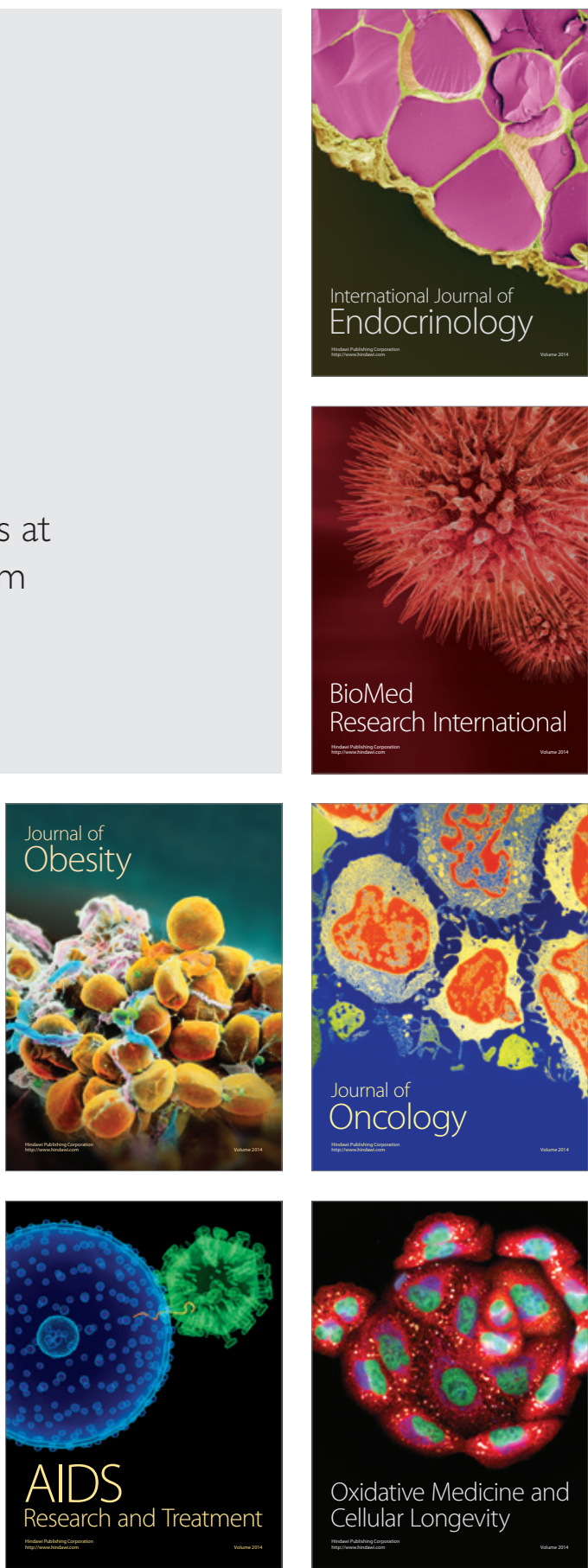